\title{
Source Density Apodisation in 2D All-Optical Ultrasound Imaging
}

\author{
Erwin J. Alles*, Sacha Noimark*, $\dagger$, Efthymios Maneas*, Wenfeng Xia*, \\ Edward Z. Zhang*, Paul C. Beard*, Ivan P. Parkin ${ }^{\dagger}$ and Adrien E. Desjardins* \\ ${ }^{*}$ Department of Medical Physics \& Biomedical Engineering, University College London, London, United Kingdom \\ ${ }^{\dagger}$ Materials Chemistry Research Centre, Department of Chemistry, University College London, London, United Kingdom \\ Email: e.alles@UCL.ac.uk
}

\begin{abstract}
In this work, an all-optical ultrasound imaging system that is capable of synthesising arbitrary source aperture geometries is presented. This capability is achieved by delivering focussed excitation light onto a spatially extended generating surface, where ultrasound is generated photoacoustically. Using a scanning mirror, the position of the resulting acoustical source was continuously varied to scan an aperture. This system exhibited sufficient sensitivity to acquire $2 \mathrm{D}$ images of clinically relevant tissue in under a second, as demonstrated on a tissuemimicking phantom. The flexibility in the source array geometry was demonstrated through the implementation of two source array geometries on the same system, which allowed for the direct comparison of the image quality. It was shown that applying source density apodisation to obtain an aperiodic source array resulted in an improvement of up to $5 \mathrm{~dB}$ in image contrast, as compared to using a conventional, periodic array exhibiting the same number of sources and spatial extents.
\end{abstract}

Index Terms-All-optical ultrasound, aperiodic arrays, spatial apodisation

\section{INTRODUCTION}

Conventional, electronic ultrasound imaging probes typically comprise a multitude of piezoelectric or capacitive transducers, which are arranged periodically and have fixed positions due to manufacturing constraints. The finite spatial extents of such arrays lead to side lobes that deteriorate the image quality. To suppress these artefacts, amplitude apodisation is commonly applied [1] to reduce the influence of transducer elements close to the array edges. With high frequency probes, which typically contain transducer elements exceeding the Nyquist limit (i.e., elements have widths larger than half the shortest wavelength), array periodicity results in grating lobe artefacts [2] that are challenging to suppress. Furthermore, piezoelectric and capacitive transducers exploit mechanical resonance to improve sensitivity; the centre frequency and bandwidth of a transducer element are hence determined by the its geometry. Consequently, the spatial resolution and penetration depth of an electronic ultrasound probe are fixed.

Recently, all-optical ultrasound imaging has developed into an attractive alternative to conventional ultrasound technology [3]-[5]. With this modality, the photoacoustic effect is

This work was supported by an ERC Starting Grant [310970 MOPHIM], the EU project FAMOS [FP7 ICT, Contract 317744], the Innovative Engineering for Health award by the Wellcome Trust [WT101957] and the EPSRC [NS/A000027/1], the EPSRC funded UCL Centre for Doctoral Training in Medical Imaging [EP/L016478/1], and the Ramsay Memorial Trust. utilised to convert excitation light within an optically absorbing structure into pressure fields that propagate through the surrounding tissue or medium. These pressure fields can exhibit pressures and bandwidths that rival or exceed those generated with conventional transducers [4], [6]. Not relying on mechanical resonance effects, temporal modulation of the excitation light can be employed to tune an optical ultrasound source to low or high frequencies, as well as narrow or wide bandwidths, to optimise the source for specific applications [7].

In this work, an imaging system that uses a spatially extended optical ultrasound generator in conjunction with a focussing lens and scanning mirror is presented. Optical spatial confinement rather than mechanical separation (as typically applied in electronic transducer arrays) is used to determine the source dimensions; as such, an optical acoustic source can be positioned arbitrarily across the ultrasound generator. By sequentially delivering excitation light to different positions, an acoustic source aperture of arbitrary geometry can be synthesised, which may even contain spatially overlapping sources to reduce the interelement pitch. Here, this flexibility in array geometry is demonstrated by directly comparing the image quality obtained using both a conventional, periodic source array and a novel aperiodic array in which the source density is given by a commonly applied apodisation window. The performance of the system for both arrays is demonstrated on both a wire phantom and a tissue mimicking phantom.

\section{Methods}

\section{Optical ultrasound generation}

A pulsed laser (wavelength: $1064 \mathrm{~nm}$, pulse duration: $5 \mathrm{~ns}$, pulse energy: $50 \mu \mathrm{J}$, pulse repetition rate: $1 \mathrm{kHz}$, beam diameter: $1.0 \mathrm{~mm}$; FQS-400-1-Y-1064, Elforlight, U.K.) was used to generate ultrasound in an optically absorbing surface comprising multi-walled carbon nanotubes and polydimethylsiloxane (PDMS) [4]. Light was spatially confined using a focussing lens (focal length: $50 \mathrm{~mm}$; resulting acoustical source diameter: $200 \mu \mathrm{m}$ ) and steered across a linear aperture using a set of scanning mirrors (GVSM002, Thorlabs, Germany). Each light pulse was delivered to a different position on the generating surface; thus a linear acoustic aperture was sequentially scanned (Fig. 1). 


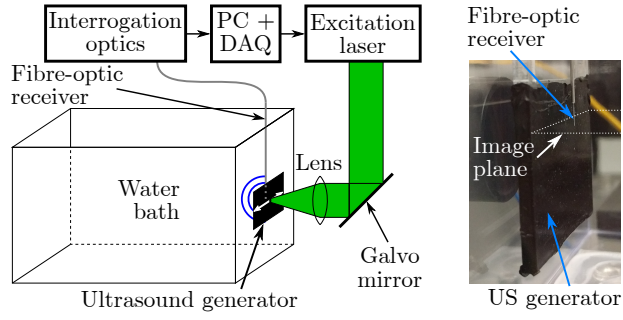

Fig. 1. Schematic (left) and photograph (right) of the all-optical ultrasound imaging setup. Pulsed excitation light was focussed onto an optically absorbing structure, where it was photoacoustically converted into an ultrasound field. Using a galvo mirror, an acoustic source aperture was sequentially synthesised. Back-scattered acoustic waves were recorded using an optical receiver comprising a Fabry-Pérot cavity fabricated on the tip of an optical fibre, which was interrogated using a wavelength tuneable laser. The horizontal image plane is indicated with white dashed lines.

\section{Optical ultrasound detection}

A broadband, highly sensitive and nearly omni-directional custom fibre-optic ultrasound detector [8] comprising a highfinesse Fabry-Pérot cavity fabricated on the tip of an optical fibre was used to record back-scattered acoustic waves. This detector was laterally centered and placed $1 \mathrm{~mm}$ away from the ultrasound generator, and interrogated by measuring the cavity's reflectivity using a continuous-wave tunable laser (TUNICS T100S-HP, Yenista, France) and a broadband photodiode (DET01CFC, Thorlabs, Germany) [9]. The reflectivity signal was amplified (+60 dB; DHPVA-200, Femto, Germany) and sampled (125 MSa/s, 14-bit; M4i.4420-x8, Spectrum, Germany) without signal averaging. The experimental setup is shown in Fig. 1. A custom LabVIEW script (LabVIEW 2014, National Instruments, TX, USA) was used to control the experimental setup.

\section{Signal processing and image reconstruction}

Recorded pulse-echo A-scans were band-pass filtered (2 $15 \mathrm{MHz}$ ), and cross-talk resulting from acoustic waves propagating directly from the generator to the detector was suppressed through temporal windowing. A delay-and-sum algorithm [1] was used to reconstruct the B-scan data into an image after applying power-law time-gain compensation. The reconstructed images measured $16 \mathrm{~mm} \times 8 \mathrm{~mm}$ at a pixel size of $10 \mu \mathrm{m} \times 10 \mu \mathrm{m}$.

\section{Array geometry and apodisation}

Two source array geometries were tested. First, a conventional, periodic array comprising 128 sources equidistantly distributed across a linear aperture with a width of $15.5 \mathrm{~mm}$ was tested, which resulted in a uniform inter-element pitch of $119 \mu \mathrm{m}$. Due to its dimensions and transducer periodicity, this array suffered from both side and grating lobes. Second, an aperiodic array was tested, in which the spatial density of the 128 sources was given by the Hamming apodisation window $W$,

$$
W(i)=0.54-0.46 \cos \left(2 \pi \frac{i-1}{N-1}\right),
$$

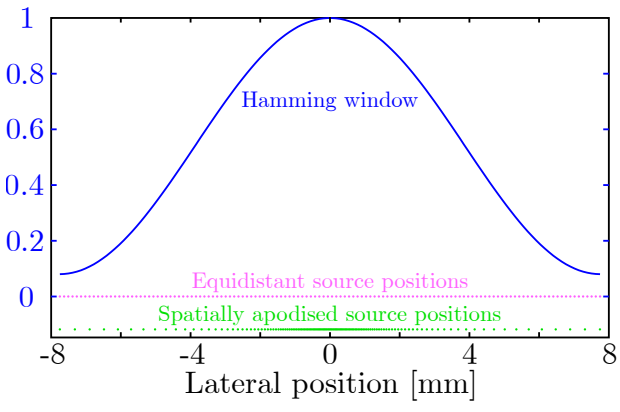

Fig. 2. A Hamming window (blue) was used for both amplitude and source density apodisation. The positions of the sources in the periodic (pink) and source density apodised arrays (green) are indicated underneath.

where $1 \leq i \leq N$ is the current source number and $N$ the total number of sources. The source positions were obtained through a process dubbed "source density apodisation". With this process, the source positions were computed by numerical integration of the reciprocal of the spatial source density, followed by normalisation and scaling to the same aperture width of $15.5 \mathrm{~mm}$. The inter-element pitch of the resulting aperiodic source array ranged from $35.6 \mu \mathrm{m}$ to $444 \mu \mathrm{m}$. Compared to a conventional periodic source array using the same number of sources and aperture size, both side and grating lobes are expected to be suppressed using this aperiodic array.

Images were reconstructed from data obtained with both source array geometries, both in the absence and in the presence of additional Hamming amplitude apodisation. Amplitude apodisation was applied by multiplying each $i$-th A-scan with the corresponding weight $W(i)$ (given by Eq. 1) interpolated to the $i$-th source position. The Hamming apodisation window $W$ and the positions of the 128 sources of both the periodic and source density apodised arrays are displayed in Fig. 2.

\section{Phantoms}

Two phantoms were used in this work. The first phantom, consisting of two layers of parallel tungsten wires (wire diameter: $27 \mu \mathrm{m}$, inter-wire spacing: $1 \mathrm{~mm}$ ) separated by a distance of $3 \mathrm{~mm}$, was used to study the effect of amplitude and source density apodisation on the image quality. A second phantom, fabricated using a tissue-mimicking material based on mineral oil and glass micro spheres [10], was used to demonstrate the feasibility of imaging clinically relevant tissues. This second phantom was designed to emulate part of the vasculature of a human placenta, and was scanned using the source density apodised array.

\section{RESULTS}

Applying Hamming amplitude apodisation to a periodic array resulted in a visible reduction in image artefacts (Fig. 3), thus confirming how amplitude apodisation can effectively suppress side lobes. However, when only source density apodisation was applied, a further reduction in artefact level was observed. The best image contrast was obtained when both source density and amplitude apodisation were applied. In this latter case, the image contrast was improved by up 

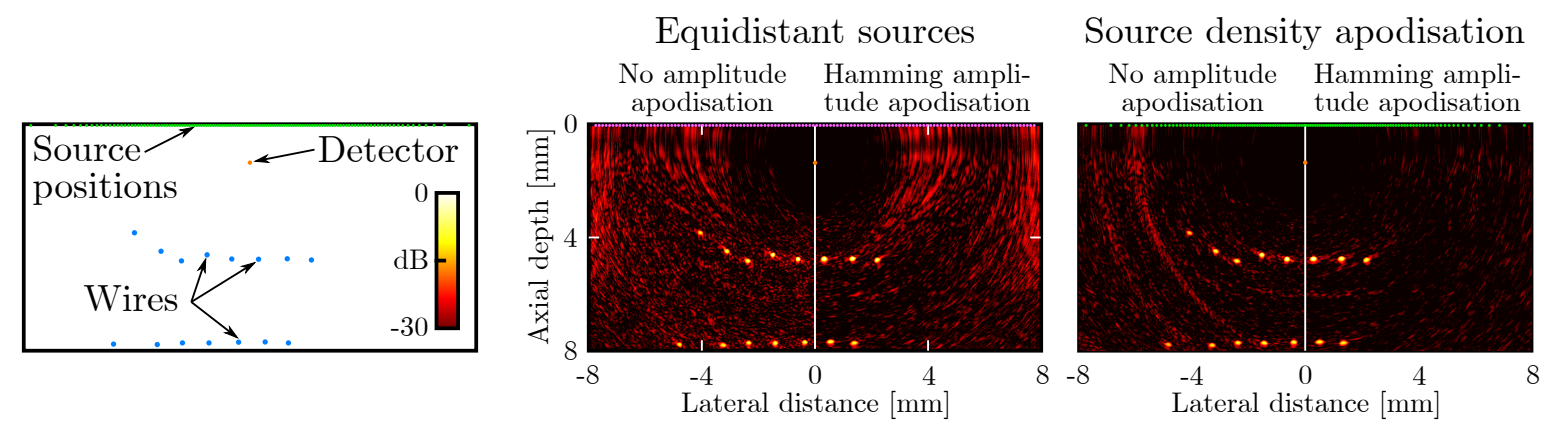

Fig. 3. Schematic (left) and all-optical ultrasound images (middle, right) of a phantom consisting of two layers of wires. Images were obtained using either a periodic (middle) or source density apodised array (right), both in the absence (left-hand-sides) and presence (right-hand-sides) of additional amplitude apodisation. All images are shown on a logarithmic scale at a dynamic range of $30 \mathrm{~dB}$.

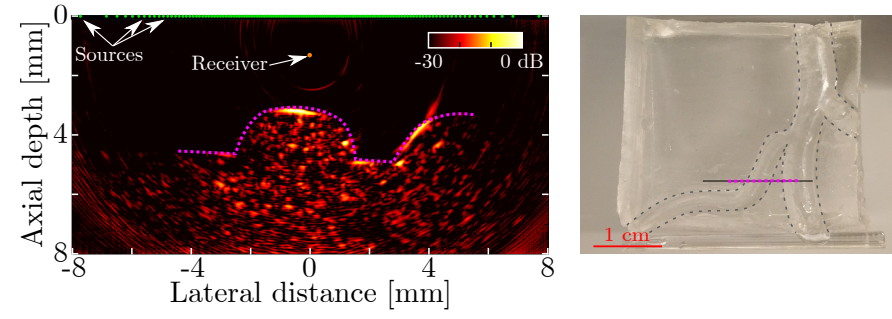

Fig. 4. All-optical ultrasound image (left) and photograph (right) of a tissuemimicking phantom emulating placental vasculature (indicated in gray dashed curves). The image plane is indicated by the solid black line; the highlighted structures by the dotted purple curves.

to $5 \mathrm{~dB}$ compared to that obtained using a periodic source array in the absence of amplitude apodisation, resulting in a dynamic range of $30 \mathrm{~dB}$ that was virtually artefact free. A slight reduction in resolution was observed when either amplitude apodisation, source density apodisation, or both apodisation schemes are applied. This trade-off between image artefact levels and resolution was previously reported [2], and is commonly observed when amplitude apodisation is applied.

Applying only source density apodisation consistently yielded better image contrast, as compared to applying only amplitude apodisation to periodic arrays, regardless of the number of sources used (data not shown). In addition, it was found that applying both apodisation schemes to an aperiodic array resulted in image contrasts similar to those obtained using a periodic array containing twice the number of sources; thus, the frame-rate could effectively be doubled by switching from a periodic source array to a source density apodised array.

Experiments on a tissue-mimicking phantom confirmed that the all-optical ultrasound imaging setup presented here has sufficient sensitivity to yield images at a dynamic range of $30 \mathrm{~dB}$ (Fig. 4). At this dynamic range, both the surface and subsurface speckle were clearly visualised up to a depth of $8 \mathrm{~mm}$.

\section{Discussion AND CONCLUSION}

The all-optical ultrasound imaging system presented in this study was capable of acquiring $2 \mathrm{D}$ images of a tissue mimicking phantom in under one second, whereas previously reported systems required minutes to hours [3]-[5], [11]. This reduction in acquisition speed was accomplished through the combination of an efficient optical ultrasound generator, an exquisitely sensitive optical ultrasound detector, and spatially overlapping acoustic sources. Using a diode-pumped solid state laser, pulses were generated at modest pulse energies and $\mathrm{kHz}$-range repetition rates, with a beam quality well suited to scanning. The use of a spatially extended optical ultrasound generator, combined with a focussing lens and scanning mirror, allowed for multiple, arbitrary source array geometries to be realised on the same setup. Using source density apodisation to obtain an aperiodic source array that was free from grating lobes and exhibited significantly suppressed side lobes, a reduction in image artefact level was obtained compared to that obtained using a conventional, periodic array.

Here, only two source array geometries were considered: a periodic array to emulate the performance of a conventional, electronic imaging probe, and an aperiodic array where the source density was given by a Hamming apodisation window that is commonly applied to periodic transducer arrays. However, a wide range of apodisation schemes exists, and further research is required to determine which source density apodisation schemes yield superior performance in various contexts. Only 2D imaging was considered in this study; in future studies, source density apodisation could readily be adapted to two-dimensional arrays for 3D imaging.

In this work, an image depth of $8 \mathrm{~mm}$ was obtained in phantoms, which is similar to that obtained with intravascular ultrasound imaging [12]. This image depth was equal to the separation between the ultrasound generator and the adjacent wall of the water bath through which excitation light was delivered. While signals were received from greater depths, the corresponding part of the image was cluttered with reflection artefacts originating from the adjacent wall. However, this image depth could be improved upon through a change in optics; using a focussing lens with a longer focal distance would allow for a larger separation between the wall of the water bath and the optical ultrasound generator.

In electronic imaging probes, the transducer elements can typically operate simultaneously and in full duplex mode, allowing for beam-forming techniques that greatly enhance the 
signal-to-noise ratio of the recorded A-scans. Consequently, conventional ultrasound probes exhibit dynamic ranges of up to $60-70 \mathrm{~dB}$. The data presented here were obtained using arrays comprising 128 sources that were sequentially excited, and yielded images that were virtually free from artefacts up to a dynamic range of $30 \mathrm{~dB}$. With the all-optical setup presented here, the dynamic range could be improved by increasing the number of sources, at the expense of a decreased frame-rate. Alternatively, further improvements to the source efficiency, detector sensitivity, and source array geometry could be investigated. Finally, the simultaneous use of multiple optical acoustic detectors could further improve the dynamic range and reduce limited view artefacts, at the expense of increases in experimental cost and complexity.

This work demonstrates how optically generated ultrasound sources allow for greater flexibility in source array geometry, and how changes in the array geometry can improve the image quality or increase the frame-rate of all-optical ultrasound imaging. In addition, the source array geometry can be reconfigured during operation to tailor the system to specific imaging contexts. An absence of electronic or metal components in the optical acoustic sources and receivers confers MRI compatibility and insensitivity to electromagnetic interference, and allows for concurrent multimodality imaging. Furthermore, an absence of high-amplitude, high-frequency electrical signals such as those typically employed in electronic ultrasound imaging arrays allows for the application of optical ultrasound in electrically sensitive environments. Thus, all-optical ultrasound imaging is a flexible modality that could lead to a wide range of novel applications.

\section{REFERENCES}

[1] M. Karaman, P.-C. Li, and M. O'Donnell, "Synthetic aperture imaging for small scale systems," Ultrasonics, Ferroelectrics, and Frequency
Control, IEEE Transactions on, vol. 42, no. 3, pp. 429-442, 1995.

[2] R. Cobbold, Foundations of Biomedical Ultrasound. New York: Oxford University Press, USA, 2007.

[3] R. Colchester, E. Zhang, C. Mosse, P. Beard, I. Papakonstantinou, and A. Desjardins, "Broadband miniature optical ultrasound probe for high resolution vascular tissue imaging," Biomedical Optics Express, vol. 6, no. 4, pp. 1502-1511, 2015.

[4] S. Noimark, R. Colchester, B. Blackburn, E. Zhang, E. Alles, S. Ourselin, P. Beard, I. Papakonstantinou, I. Parkin, and A. Desjardins, "Carbon-nanotube-PDMS composite coatings on optical fibres for alloptical ultrasound imaging," Advanced Functional Materials, vol. 26, no. 35,2016

[5] E. Alles, N. Fook Sheung, S. Noimark, E. Zhang, P. Beard, and A. Desjardins, "A reconfigurable all-optical ultrasound transducer array for 3D endoscopic imaging," Scientific Reports, vol. 7, 2017.

[6] Y. Hou, J.-S. Kim, S.-W. Huang, S. Ashkenazi, L. Jay Guo, and M. O'Donnell, "Characterization of a broadband all-optical ultrasound transducer-from optical and acoustical properties to imaging," Ultrasonics, Ferroelectrics, and Frequency Control, IEEE Transactions on, vol. 55, no. 8, pp. 1867-1877, 2008.

[7] E. Alles, R. Colchester, and A. Desjardins, "Adaptive light modulation for improved resolution and efficiency in all-optical pulse-echo ultrasound," Ultrasonics, Ferroelectrics, and Frequency Control, IEEE Transactions on, vol. 63, no. 1, pp. 83-90, 2016.

[8] E. Zhang and P. Beard, "Characteristics of optimized fibre-optic ultrasound receivers for minimally invasive photoacoustic detection," in Proceedings SPIE BiOS. International Society for Optics and Photonics, 2015, Conference Proceedings, pp. 932 311-932311-9.

[9] E. Zhang, J. Laufer, and P. Beard, "Backward-mode multiwavelength photoacoustic scanner using a planar Fabry-Pérot polymer film ultrasound sensor for high-resolution three-dimensional imaging of biological tissues," Applied Optics, vol. 47, no. 4, pp. 561-577, 2008.

[10] E. Maneas et al., "Anatomically realistic ultrasound phantoms using gel wax with 3D printed moulds," Physics in Medicine and Biology, vol. Under review, 2017.

[11] J. Johnson, J. Shragge, and K. van Wijk, "Nonconfocal all-optical laser-ultrasound and photoacoustic imaging system for angle-dependent deep tissue imaging," Journal of Biomedical Optics, vol. 22, no. 4, pp. 041 014-041 014, 2017.

[12] S. Nissen and P. Yock, "Intravascular ultrasound," Circulation, vol. 103, no. 4, pp. 604-616, 2001. 